\title{
Biological effects of NODAL on endometrial cancer cells and its underlying mechanisms
}

\author{
XIAOSHAN HONG $^{1 *}$, BIN WEN $^{1 *}$, HUAMING ZHANG $^{1}$, YUHAN LI $^{1}$, \\ HENGYING WU ${ }^{1}$, WEI ZHAO ${ }^{2}$ and XIPING LUO ${ }^{1}$

\begin{abstract}
${ }^{1}$ Department of Gynecology, Guangdong Women and Children Hospital, Guangzhou, Guangdong 510010;
${ }^{2}$ Guangdong Provincial Key Laboratory of Medical Molecular Diagnostics, Institute of Aging Research, Guangdong Medical University, Dongguan, Guangdong 523808, P.R. China
\end{abstract}

Received March 12, 2020; Accepted August 13, 2020

DOI: $10.3892 /$ etm.2021.9833

\begin{abstract}
Activin A receptor type 1C (ALK7) and its ligand nodal growth differentiation factor (NODAL) serve numerous roles in cancer cells, including regulating cancer invasion, migration and apoptosis. NODAL promotes breast cancer cell apoptosis by activating ALK7; however, ALK7 and NODAL expression in endometrial cancer (EC), as well as their effects and underlying mechanisms in EC cells, are not completely understood. The present study aimed to characterize the expression of NODAL and ALK7 in EC, as well as the underlying mechanisms. The expression levels of ALK7 and NODAL were detected via reverse transcription-quantitative PCR and western blotting. Cell transfection was performed to overexpress NODAL or interfere ALK7. Cell proliferation, invasion and migration were detected via Cell Counting Kit-8, Transwell and wound healing assays, respectively. Flow cytometry was performed to detect cell apoptosis and western blotting was conducted to detect the expression levels of apoptosis-related proteins. NODAL and ALK7 expression levels were significantly decreased in EC cell lines compared with normal endometrial cells. NODAL overexpression inhibited EC cell proliferation, invasion and migration, and promoted EC cell apoptosis compared with the overexpression-negative control (Ov-NC) group. Moreover,
\end{abstract}

Correspondence to: Dr Xiping Luo, Department of Gynecology, Guangdong Women and Children Hospital, 521 Xingnan Avenue, Panyu, Guangzhou, Guangdong 510010, P.R. China

E-mail: luoxping333@126.com

Dr Wei Zhao, Guangdong Provincial Key Laboratory of Medical Molecular Diagnostics, Institute of Aging Research, Guangdong Medical University, 1 Xincheng Road, Dongguan, Guangdong 523808, P.R. China

E-mail: davidzhao1999@foxmail.com

*Contributed equally

Key words: endometrial cancer, nodal growth differentiation factor, activin A receptor type $1 \mathrm{C}$, invasion, migration, apoptosis
NODAL overexpression significantly increased ALK7 expression levels in EC cells compared with the Ov-NC group. ALK7 reversed NODAL overexpression-mediated inhibition of EC cell proliferation, invasion and migration, and promotion of EC cell apoptosis. The present study indicated that NODAL inhibited EC cell proliferation, invasion and migration, and promoted EC cell apoptosis by activating ALK7.

\section{Introduction}

Endometrial cancers (ECs) are a group of epithelial malignant tumors that occur in the endometrium, accounting for 20-30\% of all malignancies of the female genital tract (1). The average age of onset of EC is 60 years and $75 \%$ of EC cases occur in women aged $>50$ years (2). At present, EC treatment is primarily based on surgery supplemented with radiotherapy, chemotherapy and hormone therapy (3). However, due to the side effects of radiotherapy and chemotherapy, patients suffer greatly (3). Therefore, the aim of the present study was to identify novel therapeutic targets to provide potential new treatment strategies for EC.

Nodal growth differentiation factor (NODAL) is an important member of the TGF- $\beta$ family and an important morphogenetic molecule in embryonic development (4). Previously, it was thought that NODAL was only expressed in embryonic tissues (5); however, in 2006, Topczewska et al (6) reported that NODAL is highly expressed in melanoma and is closely associated with occurrence and metastasis, serving a protumor effect. Since then, abnormal expression of NODAL has been identified in breast, prostate, pancreatic, liver and other types of cancer (7-10). Additionally, studies have reported that NODAL promotes tumor development $(11,12)$; however, other studies have indicated that NODAL inhibits tumor cell proliferation and promotes apoptosis, as evidenced by dose-dependent studies on prostate and pancreatic cancer $(13,14)$. To the best of our knowledge, the role of NODAL in EC has not been previously reported.

Activin receptor-like kinase 7 (ALK7) is one of seven type I receptors in the TGF- $\beta$ family (15). A previous study demonstrated that the TGF- $\beta$ family members NODAL, Activin A/B and growth differentiation factor 3 are all ligands of ALK7 (16). Compared with NODAL, few studies have 
investigated ALK7 and the studies on its function primarily focused on two aspects. Firstly, ALK7 has been reported to inhibit cell proliferation and promote cell apoptosis following activation (17). Secondly, ALK7 is involved in sugar and lipid metabolism (18). Furthermore, a recent study has demonstrated that ALK7, as a tumor suppressor gene, is a barrier to tumor occurrence and metastasis (19). However, to the best of our knowledge, the expression, effect and underlying mechanisms of ALK7 in EC are not completely understood.

NODAL is the ligand of ALK7 and promotes breast cancer cell apoptosis via ALK7 (20). Bioinformatics analysis (ualcan.path.uab.edu) indicated that the expression levels of NODAL and ALK7 in EC cell lines were decreased. However, the expression of NODAL and ALK7 in EC and their effects on tumor cell proliferation, invasion and migration are not completely understood. Therefore, the present study investigated the effects of NODAL and ALK7 on EC cell proliferation, invasion, migration and apoptosis, as well as the underlying mechanisms, to identify novel therapeutic targets for EC.

\section{Materials and methods}

Cell culture and treatment. EC cell lines (Ishikawa, KLE, RL95-2 and AN3 CA) and a novel immortalized human endometrial stromal cell line (THESCs; referred to as ESC in the present study; RRID: CVCL_C464; NCBI Taxonomy: 9606) were purchased from The Cell Bank of Type Culture Collection of the Chinese Academy of Sciences. Cells were cultured in DMEM (Gibco; Thermo Fisher Scientific, Inc.) supplemented with $10 \%$ FBS (Gibco; Thermo Fisher Scientific, Inc.) at $37^{\circ} \mathrm{C}$ with $5 \% \mathrm{CO}_{2}$. Solid SB431542 (cat. no. HY-10431) was obtained from MedChemExpress and was dissolved in $1 \mathrm{ml}$ DMEM (Gibco; Thermo Fisher Scientific, Inc.) at a final concentration of $10 \mathrm{mmol} / \mathrm{l}$. The induction time of SB431542 is $24 \mathrm{~h}$ at $37^{\circ} \mathrm{C}$ with $5 \% \mathrm{CO}_{2}$.

Reverse transcription-quantitative PCR (RT-qPCR). Total RNA was extracted from cells using RNAzol RT (Sigma-Aldrich; Merck KGaA), according to the manufacturer's protocol. RNA concentration and quantification were assessed using a NanoDrop spectrophotometer (Thermo Fisher Scientific, Inc.). Following DNase I digestion, total RNA was reverse transcribed into cDNA using a QuantiTect Reverse Transcription kit (Qiagen $\mathrm{GmbH}$ ), according to the manufacturer's protocol. Subsequently, qPCR was performed using a QuantiTect SYBR Green PCR kit (Qiagen GmbH), according to the manufacturer's protocol. The following thermocycling conditions were used for qPCR: $95^{\circ} \mathrm{C}$ for $10 \mathrm{~min}$; followed by 40 cycles of $95^{\circ} \mathrm{C}$ for $10 \mathrm{sec}$ and $60^{\circ} \mathrm{C}$ for $60 \mathrm{sec}$. The following primers (GenScript) were used for qPCR: ALK7 forward, 5'-ATGACCCCAGCGCGCGGC TCCGCACT-3' and reverse, 5'-CTTCCTGTATGTGCA CTGGCGGTCCT-3'; NODAL forward, 5'-ACCGAGTCC CTTCCACTTGT-3' and reverse, 5'-CAGAGGCACCCA CATTCTTC-3'; and GAPDH forward, 5'-AGCCACATC GCTCAGACAC-3' and reverse, 5'-GCCCAATACGACCAA ATCC-3'. mRNA expression levels were quantified using the $2^{-\Delta \Delta \mathrm{Cq}}$ method (21) and normalized to the internal reference gene GAPDH.
Bioinformatics website. The expression levels of NODAL and ALK7 in EC were predicted using a bioinformatics website (ualcan.path.uab.edu; release date, 03/13/2019).

Western blotting. Cells were washed twice with cold PBS, lysed with RIPA lysis buffer (Beyotime Institute of Biotechnology) and incubated for $30 \mathrm{~min}$ on ice. Cell lysates were centrifuged at $300 \mathrm{x} \mathrm{g}$ at $4^{\circ} \mathrm{C}$ for $20 \mathrm{~min}$ and the protein supernatant was transferred into Eppendorf tubes. Total protein was quantified using a BCA protein assay kit (Bio-Rad Laboratories, Inc.). Proteins $(40 \mu \mathrm{g})$ were separated via $10 \%$ SDS-PAGE and transferred to PVDF membranes (GE Healthcare), which were blocked with $10 \%$ skimmed milk for $1 \mathrm{~h}$ at room temperature. Subsequently, the membranes were incubated overnight at $4^{\circ} \mathrm{C}$ with the following primary antibodies (all purchased from Abcam): Anti-NODAL (1:1,000; cat. no. ab55676), anti-ALK7 (1:1,000; cat. no. ab111121), anti-matrix metallopeptidase (MMP)2 (1:1,000; cat. no. ab215986), anti-MMP7 (1:1,000; cat. no. ab205525), anti-MMP9 (1:1,000; cat. no. ab219372), anti-caspase-3 (1:1,000; cat. no. ab13847), anti-caspase-9 (1:1,000; cat. no. ab65608), anti-cleaved-caspase-3 (1:1,000; cat. no. 9953S), anti-cleaved-caspase-9 (1:1,000; cat. no. ab2324), anti-Bcl2 (1:1,000; cat. no. ab32124), anti-Bax $(1: 1,000$; cat. no. ab32503) and anti-GAPDH (1:1,000; cat. no. ab181602). Following primary incubation, the membranes were incubated with a goat anti-rabbit horseradish peroxidase-conjugated IgG secondary antibodies (1:5,000; cat. no. AA24142; Abcam) at room temperature for $1 \mathrm{~h}$. Protein bands were visualized using enhanced chemiluminescence reagent (GE Healthcare). Protein expression levels were semi-quantified using ImageJ software (version 1.46; National Institutes of Health) with GAPDH as the loading control.

Cell transfection. Cells ( $1 \times 10^{5}$ cells/well) were seeded into 6-well plates and cultured for $24 \mathrm{~h}$ at $37^{\circ} \mathrm{C}$ with $5 \% \mathrm{CO}_{2}$. Subsequently, cells were transfected with NODAL overexpression vector (Ov-NODAL), empty vector (NC; Ov-NC), two different ALK7-targeting short hairpin (sh)RNA (shRNA-ALK7-1 and shRNA-ALK7-2) or shRNA-NC at a concentration of $20 \mathrm{nM}$. All plasmids were obtained from Shanghai GenePharma Co., Ltd. and transfected using Lipofectamine ${ }^{\circledR} 3000$ (Invitrogen; Thermo Fisher Scientific, Inc.), according to the manufacturer's protocol. Cells in the blank control group (Control) were untreated. At $48 \mathrm{~h}$ post-transfection, transfection efficiency was assessed via RT-qPCR.

Cell Counting Kit-8 (CCK-8) assay. Cells ( $1 \times 10^{3}$ cells/well) were seeded into 96 -well plates and incubated at $37^{\circ} \mathrm{C}$ with $5 \% \mathrm{CO}_{2}$. Cell proliferation was determined using CCK-8 reagent (Dojindo Molecular Technologies, Inc.), according to the manufacturer's protocol. Following transfection for 24,48 or $72 \mathrm{~h}, 10 \mu \mathrm{l}$ CCK-8 solution was added to each well for $4 \mathrm{~h}$ at $37^{\circ} \mathrm{C}$. Absorbance was measured at a wavelength of $450 \mathrm{~nm}$ using a microplate reader. SB431542 was added and induced for $24 \mathrm{~h}$, followed by cell transfection. CCK- 8 experiment was performed again $72 \mathrm{~h}$ later.

Wound healing assay. Cells were seeded ( $1 \times 10^{5}$ cells/well) into 12 -well plates. At $80 \%$ confluence, the medium was replaced with serum-free DMEM and cells were incubated 
at $37^{\circ} \mathrm{C}$ overnight. Subsequently, a $200-\mu 1$ pipette tip was used to scratch the cell monolayer. Following washing with PBS to remove free-floating cells and debris, the plates were maintained at $37^{\circ} \mathrm{C}$ with $5 \% \mathrm{CO}_{2}$. Following incubation for $48 \mathrm{~h}$, the wounds were observed using a BX51 inverted microscope (Olympus Corporation; magnification, x100). Cell migration was quantified as follows: ( $0 \mathrm{~h}$ scratch width-scratch width following culturing)/0 h scratch width.

Cell invasion assay. To assess cell invasion, 24-well Transwell plates (Corning, Inc.) with $8-\mu \mathrm{m}$ pore inserts were coated with Matrigel (BD Biosciences) at $37^{\circ} \mathrm{C}$ for $30 \mathrm{~min}$. Cells $\left(5 \times 10^{4}\right.$ cells $\left./ \mathrm{ml}\right)$ in $200 \mu \mathrm{l}$ serum-free medi plated into the upper chamber and $600 \mu 1$ DMEM supplemented with $10 \%$ FBS was added to the lower chamber. Following incubation for $24 \mathrm{~h}$ at $37^{\circ} \mathrm{C}$ with $5 \% \mathrm{CO}_{2}$, non-invading cells were removed using a cotton-tipped swab. Invading cells were fixed with $4 \%$ formaldehyde for $15 \mathrm{~min}$ at $25^{\circ} \mathrm{C}$ and stained with $0.1 \%$ crystal violet solution for $30 \mathrm{~min}$ at room temperature. Invading cells in five randomly selected fields of view were observed using an inverted microscope (Olympus Corporation; magnification, $\mathrm{x} 100)$.

TUNEL assay. Cells were collected and washed three times with PBS. Following fixing with $4 \%$ paraformaldehyde at room temperature for $20 \mathrm{~min}$, the cells were washed twice with PBS. Then, $0.2 \%$ Triton X-100 was added to the cells at room temperature for $5 \mathrm{~min}$. Subsequently, $50 \mu \mathrm{l}$ TUNEL assay solution (Boehringer Mannheim) was added to the cells and incubated at $37^{\circ} \mathrm{C}$ in the dark for $60 \mathrm{~min}$. The detection solution was discarded and cells were washed three times with PBS. Subsequently, three fields of view were selected at random, each with about 300-500 cells and then 300-500 cells were sealed with anti-fluorescence quenched sealing solution for observation under a fluorescence microscope (Zeiss $\mathrm{GmbH}$ ). The available excitation wavelength range was $450-500 \mathrm{~nm}$ and the emission wavelength range was 515-565 $\mathrm{nm}$ (green fluorescence).

Statistical analysis. Data are expressed as the mean \pm standard deviation from $\geq 3$ independent experiments. Statistical analyses were performed using SPSS statistical software (version 22.0; IBM Corp.). Comparisons among multiple groups were analyzed using one-way ANOVA followed by Tukey's post hoc test. $\mathrm{P}<0.05$ was considered to indicate a statistically significant difference.

\section{Results}

NODAL and ALK7 expression levels are lower in EC cell lines. The expression levels of NODAL and ALK7 in EC were predicted using a bioinformatics website (ualcan.path. uab.edu). Compared with normal tissues, NODAL and ALK7 expression levels were significantly decreased in EC (Fig. 1A). Subsequently, the expression levels of NODAL and ALK7 in EC cell lines were detected via RT-qPCR (Fig. 1B) and western blotting (Fig. 1C). Compared with normal endometrial cells, the expression levels of NODAL and ALK7 in EC cell lines were significantly decreased. RL95-2 cells displayed the lowest expression levels of NODAL and ALK7 among the EC cell lines. Therefore, RL95-2 cells were selected for subsequent experiments.

NODAL overexpression inhibits EC cell proliferation, invasion and migration. To evaluate the specific role of NODAL in EC cells, NODAL overexpression was performed and confirmed via RT-qPCR (Fig. 2A) and western blotting (Fig. 2B). Compared with the Ov-NC group, NODAL expression levels were significantly increased in the Ov-NODAL group. Following NODAL overexpression, CCK-8, wound healing and Transwell assays were performed to assess cell proliferation, migration and invasion, respectively. Compared with the Ov-NC group, cell proliferation (Fig. 2C), migration (Fig. 2D and E) and invasion (Fig. 2F and G) were significantly decreased in the Ov-NODAL group. Furthermore, the expression levels of invasion and migration-related proteins (MMP2, MMP7 and MMP9) were detected. The results indicated that MMP2, MMP7 and MMP9 expression levels were significantly decreased in the Ov-NODAL group compared with the Ov-NC group (Fig. 2H). The results indicated that NODAL inhibited EC cell proliferation, invasion and migration.

NODAL overexpression promotes EC cell apoptosis. Following NODAL overexpression, apoptosis was detected by performing a TUNEL assay. Compared with the Ov-NC group, cell apoptosis was markedly increased in the Ov-NODAL group (Fig. 3A). Subsequently, the expression levels of apoptosis-related proteins were detected via western blotting. Compared with the Ov-NC group, the expression levels of the antiapoptotic protein Bcl-2 were significantly decreased, whereas the expression levels of the proapoptotic proteins Bax, cleaved-caspase-3 and cleaved-caspase- 9 were significantly increased in the Ov-NODAL group. The results indicated that NODAL promoted EC cell apoptosis (Fig. 3B).

Inhibiting ALK7 reverses the inhibitory effect of NODAL overexpression on EC cell proliferation, invasion and migration. NODAL overexpression significantly increased ALK7 expression levels compared with Ov-NC (Fig. 4A), indicating that ALK7 was activated. An ALK7-targeting shRNA was constructed and transfected into EC cells and western blotting was performed to detect ALK7 expression levels (Fig. 4A). Compared with the shRNA-NC group, the expression of ALK7 was significantly decreased in the shRNA-ALK7-1 and shRNA-ALK7-2 groups. Moreover, cells were co-transfected with shRNA-ALK7 and Ov-NODAL. RT-qPCR and western blotting were performed to measure ALK7 expression levels (Fig. 4B and C). Compared with the Ov-NODAL-NC group, the expression of ALK7 was significantly lower in the Ov-NODAL-shRNA-ALK7-1 and Ov-NODAL-shRNA-ALK7-2 groups. shRNA-ALK7-1 plasmid was selected for subsequent experiments since the inhibition of ALK7 with shRNA-ALK7-1 was markedly increased compared with shRNA-ALK7-2. Subsequently, an ALK7 inhibitor (SB431542) was used and the cells were divided into the following six groups: i) Control; ii) Ov-NC; iii) Ov-NODAL; iv) Ov-NODAL-NC; v) Ov-NODAL + shRNA-ALK7; and vi) Ov-NODAL + SB431542. Following this, cell proliferation (Fig. 4D), migration (Fig. 4E and F) and invasion (Fig. 4G and $\mathrm{H}$ ) were 


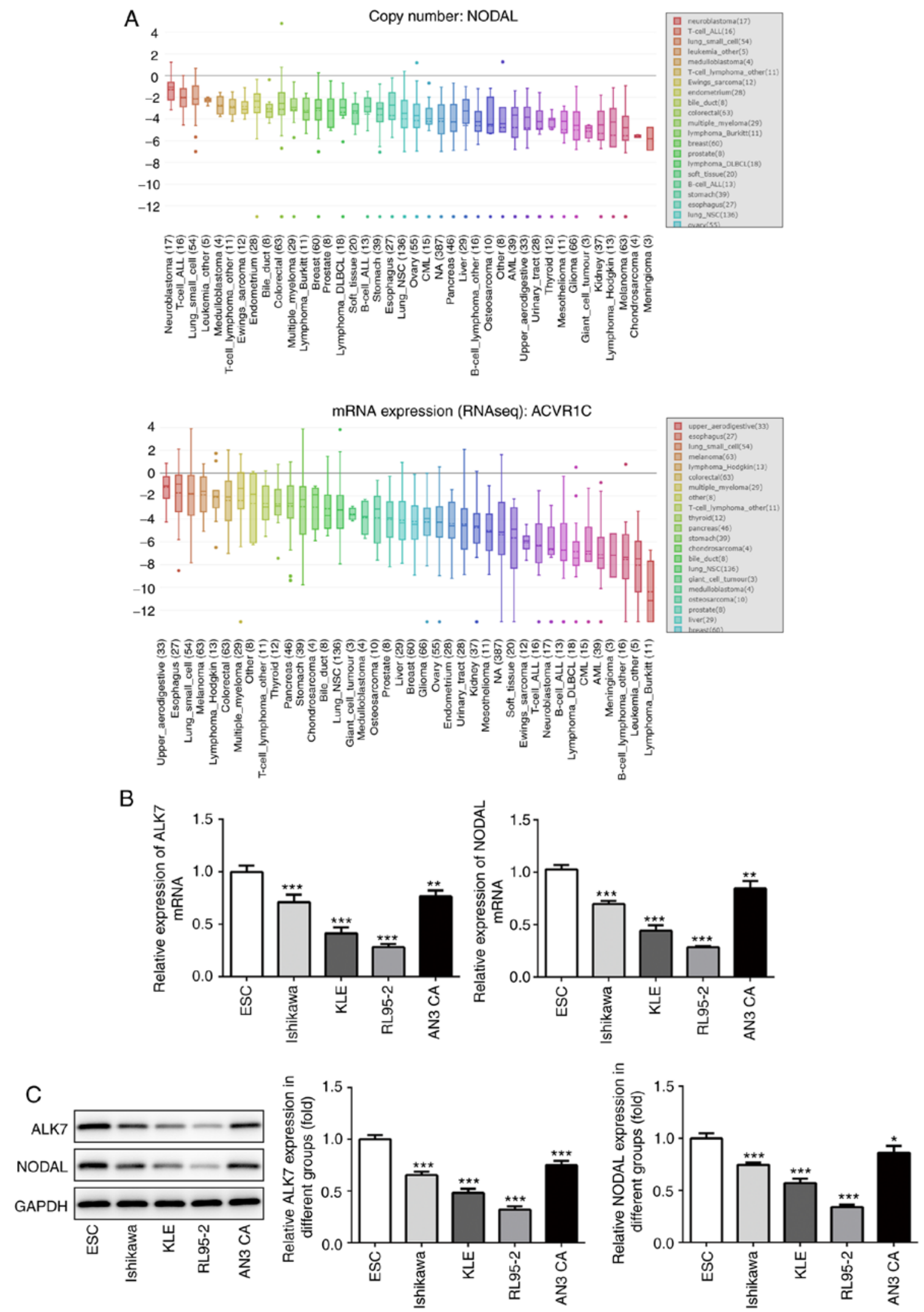

Figure 1. NODAL and ALK7 expression levels are decreased in EC cell lines. (A) Bioinformatics analysis was performed to predict the expression of NODAL and ALK7 in EC cells. ALK7 and NODAL (B) mRNA and (C) protein expression levels in EC cells. ${ }^{*} \mathrm{P}<0.05,{ }^{* * *} \mathrm{P}<0.01$ and ${ }^{* * * *} \mathrm{P}<0.001 \mathrm{vs.} \mathrm{ESC.} \mathrm{NODAL,} \mathrm{nodal}$ growth differentiation factor; ALK7, activin A receptor type 1C; EC, endometrial cancer.

assessed. Compared with the Ov-NODAL-NC group, the Ov-NODAL + shRNA-ALK7 displayed significantly increased cell proliferation, migration and invasion, and significantly upregulated expression levels of MMP2, MMP7 and MMP9 (Fig. 4I). Furthermore, compared with the Ov-NODAL group, the Ov-NODAL + SB431542 groups displayed significantly 


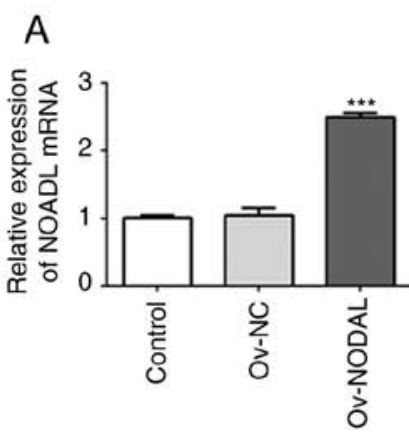

B

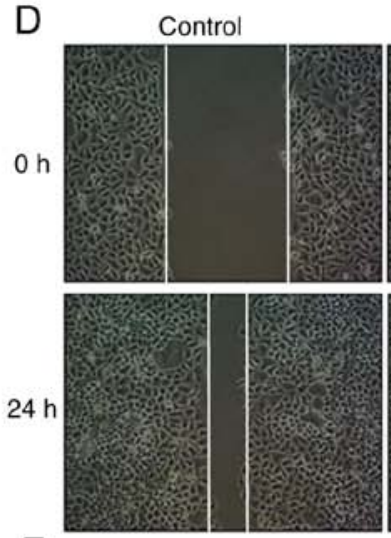

Ov-NC
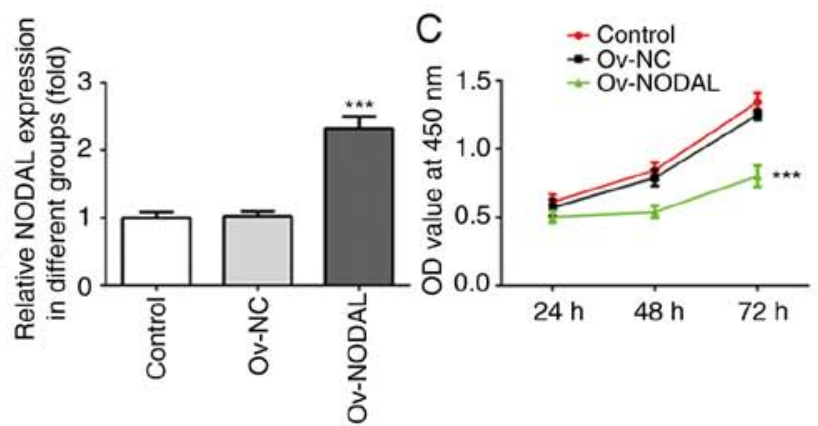

F

Control

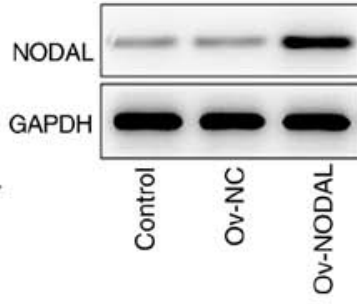

Ov-NODAL
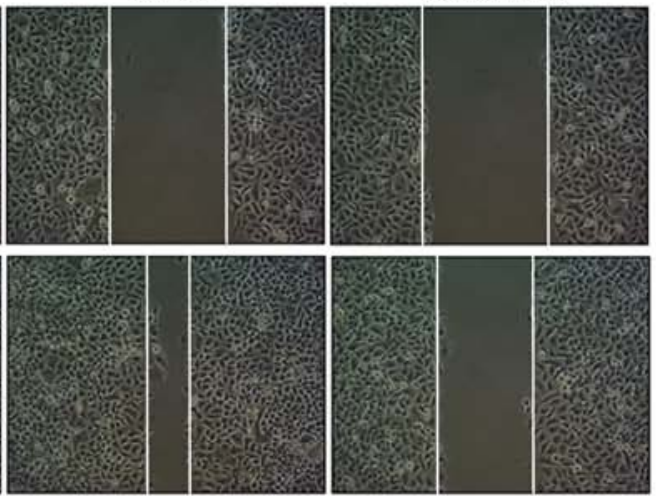

E
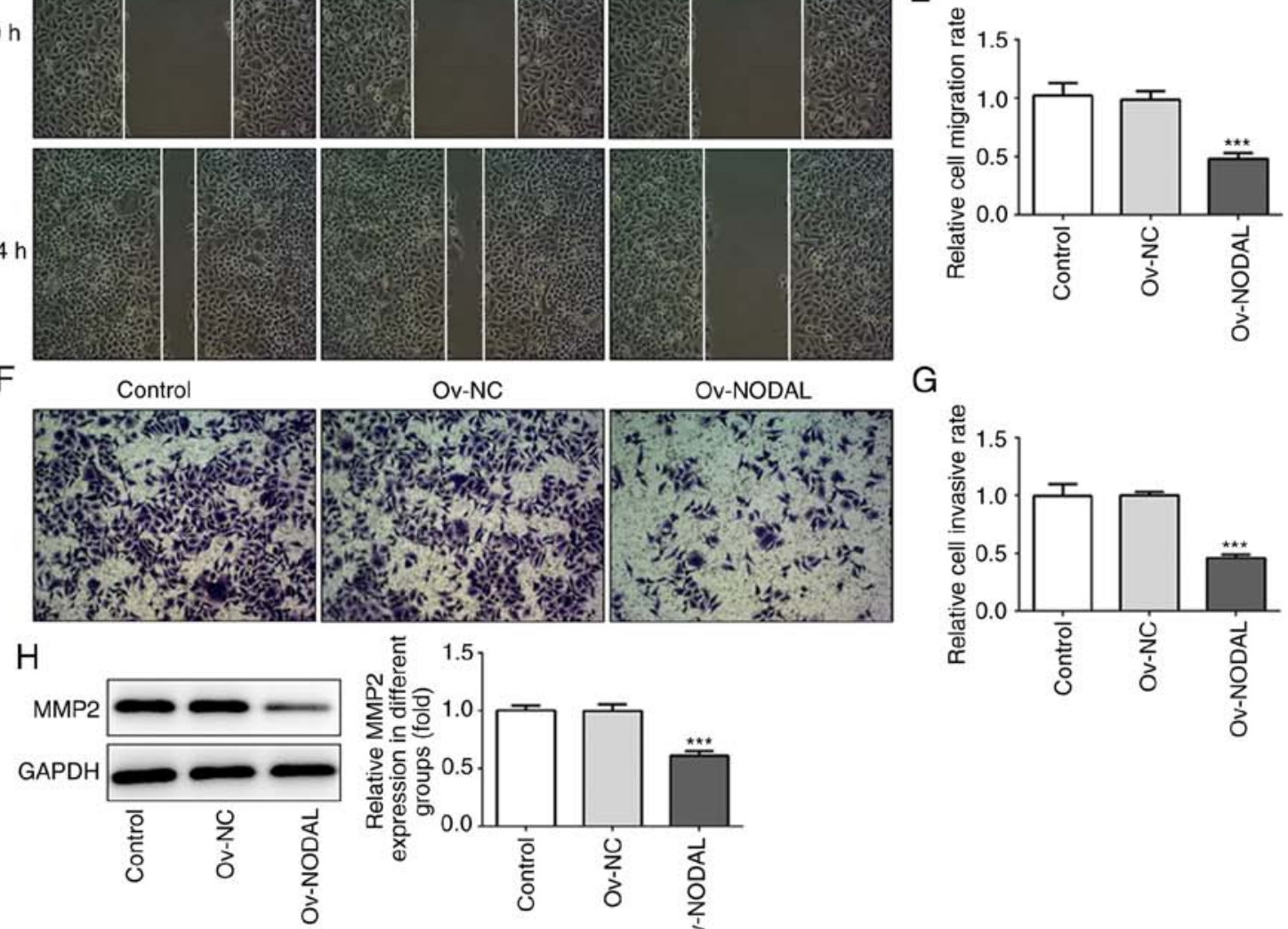

Ov-NC

Ov-NODAL

$\mathrm{G}$
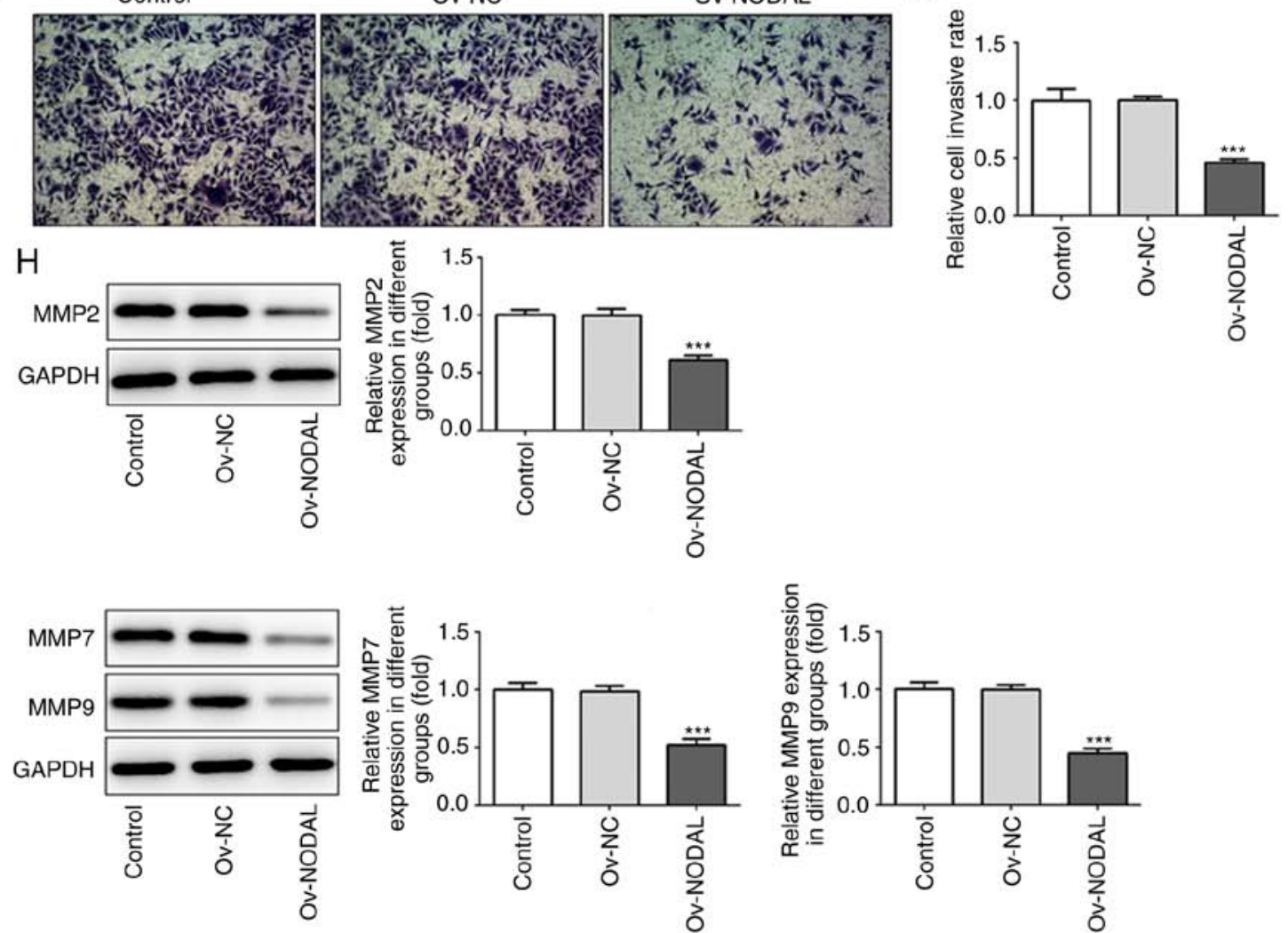

Figure 2. NODAL overexpression inhibits EC cell proliferation, invasion and migration. NODAL (A) mRNA and (B) protein expression levels following NODAL overexpression in EC cells. (C) Cell proliferation was assessed by performing a Cell Counting Kit-8 assay. (D) Representative images of the wound healing assay (magnification, x100). (E) Quantification of cell migration. (F) Representative images of the Transwell assay (magnification, x100). (G) Quantification of cell invasion. (H) MMP2, MMP7 and MMP9 protein expression levels were assessed via western blotting. ${ }^{* * *} \mathrm{P}<0.001$ vs. Ov-NC. NODAL, nodal growth differentiation factor; EC, endometrial cancer; MMP, matrix metallopeptidase; Ov, overexpression; NC, negative control; OD, optical density.

increased cell proliferation, migration and invasion, and significantly upregulated expression levels of MMP2, MMP7 and MMP9. The results suggested that NODAL overexpression activated ALK7 and inhibited EC cell proliferation, migration and invasion. Moreover, ALK7 inhibition reversed NODAL overexpression-mediated inhibition of EC cell proliferation, 
A

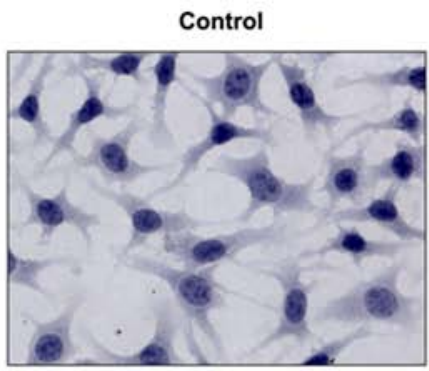

Ov-NC

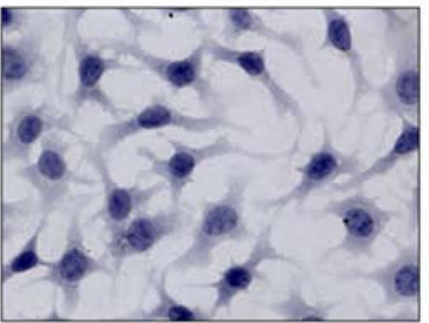

Ov-NODAL

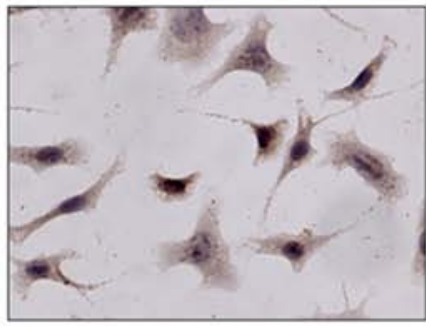

B
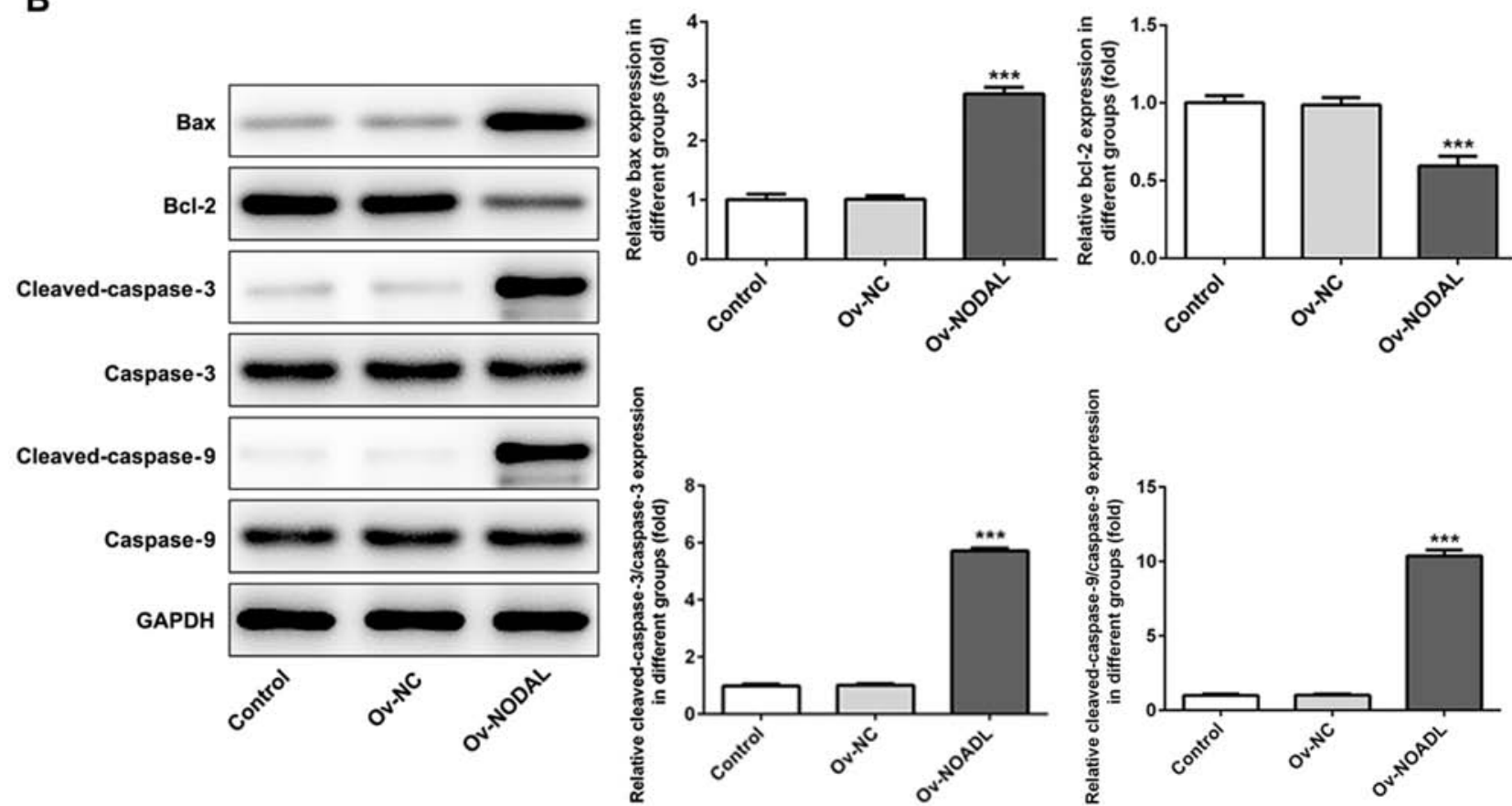

Figure 3. NODAL overexpression promotes endometrial cancer cell apoptosis. (A) Cell apoptosis was assessed by performing a TUNEL assay (magnification, $\mathrm{x} 200$ ). (B) Expression levels of apoptosis-related proteins were assessed via western blotting. ${ }^{* * *} \mathrm{P}<0.001$ vs. Ov-NC. NODAL, nodal growth differentiation factor; Ov, overexpression; NC, negative control.

migration and invasion, indicating that NODAL inhibited EC cell proliferation, invasion and migration by activating ALK7.

ALK7 inhibition reverses the effect of NODAL overexpression on EC cell apoptosis. The effect of ALK7 inhibition on cell apoptosis was assessed. Compared with the Ov-NC group, the rates of apoptosis in the Ov-NODAL + shRNA-ALK7 and Ov-NODAL + SB431542 groups were notably increased (Fig. 5A). Additionally, the expression levels of the apoptosis-related proteins Bax, cleaved-caspase-3 and cleaved-caspase-9 were significantly decreased in the Ov-NODAL-shRNA-ALK7 group compared with the Ov-NODAL-NC group (Fig. 5B). The results suggested that NODAL promoted EC cell apoptosis by activating ALK7.

\section{Discussion}

The role of NODAL in cancer has received increasing attention (22). NODAL is abnormally expressed in melanoma, participating in occurrence and metastasis (6). Later, it was reported that NODAL is abnormally expressed in glioma (23), pancreatic cancer (11), breast cancer (24) and other tumor cells, where it participates in the occurrence and development of tumors. Quail et al (25) demonstrated that the expression of NODAL is positively correlated with high vascular densities in breast lesions. Additionally, an in vitro study identified that NODAL promoted the migration of endothelial cells and vascular formation in breast cancer (25). The expression of NODAL in liver cancer tissues is increased and NODAL promotes migration, invasion and vascular formation in liver cancer cells (26). However, the expression and specific roles of NODAL in EC are not completely understood. Therefore, the present study predicted the expression of NODAL in EC using bioinformatics software, which indicated that NODAL was expressed at low levels in EC. Additionally, the in vitro cell experiments indicated that the expression of NODAL in EC cell lines was significantly decreased compared with normal endometrial cells. The present study demonstrated that NODAL overexpression inhibited EC cell proliferation, migration and invasion compared with $\mathrm{Ov}-\mathrm{NC}$, demonstrating that NODAL may inhibit tumorigenesis and development in EC.

NODAL inhibits cell proliferation and induces apoptosis in human trophoblast cells, which can be blocked by a lack of the kinase ALK7. In other words, NODAL inhibits cell proliferation and induces apoptosis by activating ALK7 (27). 
A
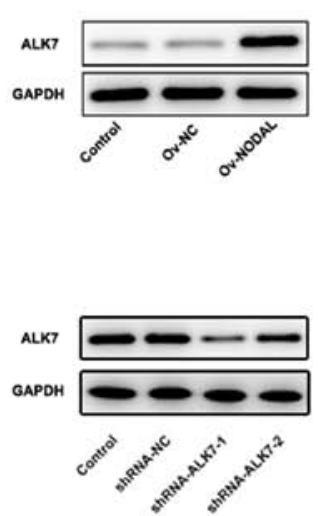

c

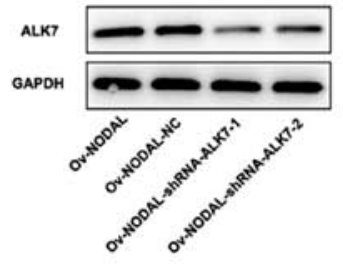

OW-NC

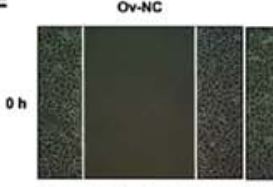

OV-NODAL

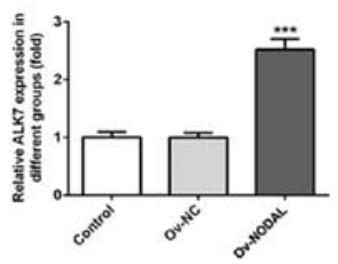

B
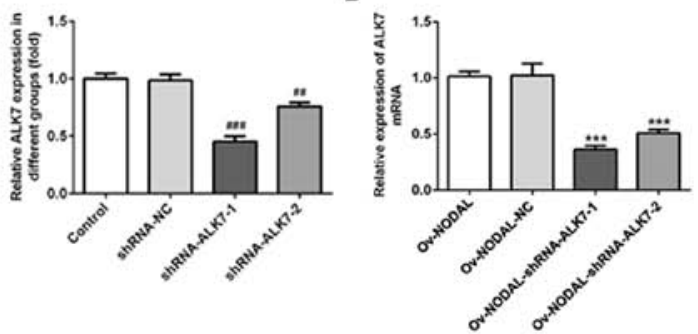

D
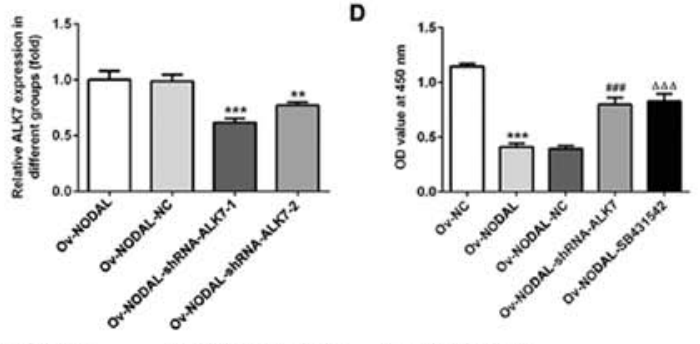

Or-NOOALSBA31542

OV-NODAL-NC

OV-NODAL-ShRNA-ALK7
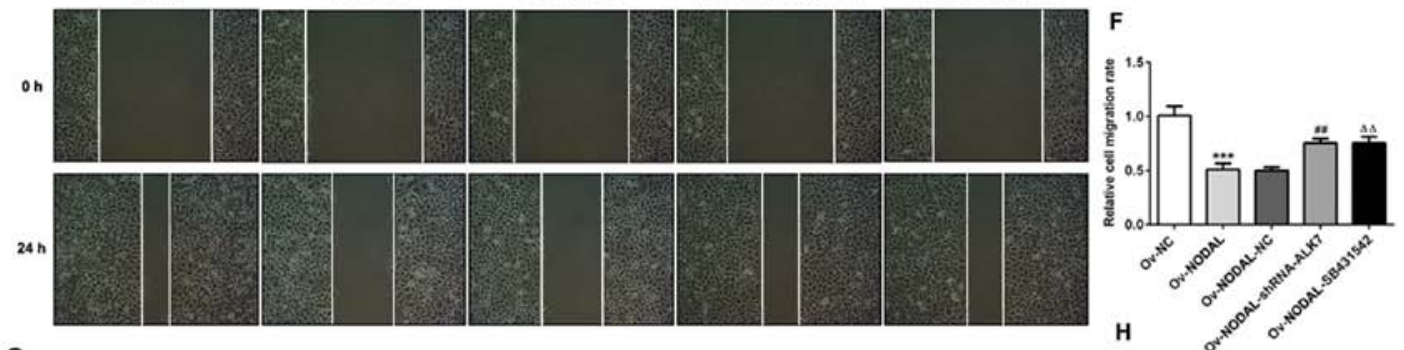

G

Ov-NC Ov-NODAL OV-NODAL-NC OV-NODAL-SHRNA-ALKT

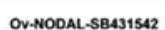

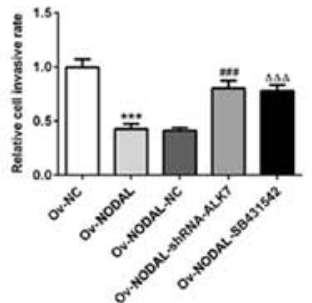

I
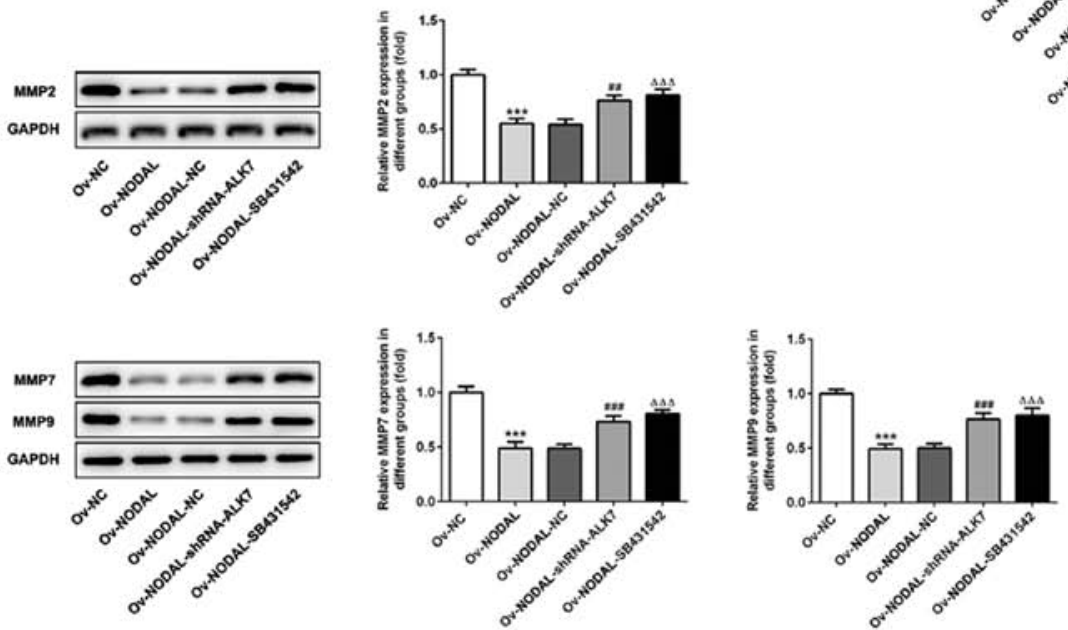

Figure 4. ALK7 inhibition reverses NODAL overexpression-mediated effects on endometrial cancer cell proliferation. (A and B) ALK7 protein expression levels in transfected cells were measured via western blotting. ${ }^{* * * *} \mathrm{P}<0.001$ vs. Ov-NC; ${ }^{\# \#} \mathrm{P}<0.01,{ }^{\# \# \#} \mathrm{P}<0.001$ vs. shRNA-NC. (C) ALK7 expression levels in co-transfected cells were measured via reverse transcription-quantitative PCR. ${ }^{* *} \mathrm{P}<0.01$ and ${ }^{* * *} \mathrm{P}<0.001$ vs. Ov-NODAL-NC. (D) Cell proliferation was assessed by performing Cell Counting Kit-8 assays. (E) Representative images of the wound healing assay (magnification, x100). (F) Quantification of cell migration. (G) Representative images of the Transwell assay (magnification, x100). (H) Quantification of cell invasion. (I) MMP2, MMP7 and MMP9 protein expression levels were measured via western blotting. ${ }^{* * *} \mathrm{P}<0.001$ vs. Ov-NC; ${ }^{\# \#} \mathrm{P}<0.01$ and ${ }^{\# \# \#} \mathrm{P}<0.001$ vs. Ov-NODAL-NC; ${ }^{\Delta \Delta} \mathrm{P}<0.01$ and ${ }^{\Delta \Delta \Delta} \mathrm{P}<0.001$ vs. Ov-NODAL. ALK7, activin A receptor type 1C; NODAL, nodal growth differentiation factor; Ov, overexpression; NC, negative control; MMP, matrix metallopeptidase; shRNA, short hairpin RNA; OD, optical density. 


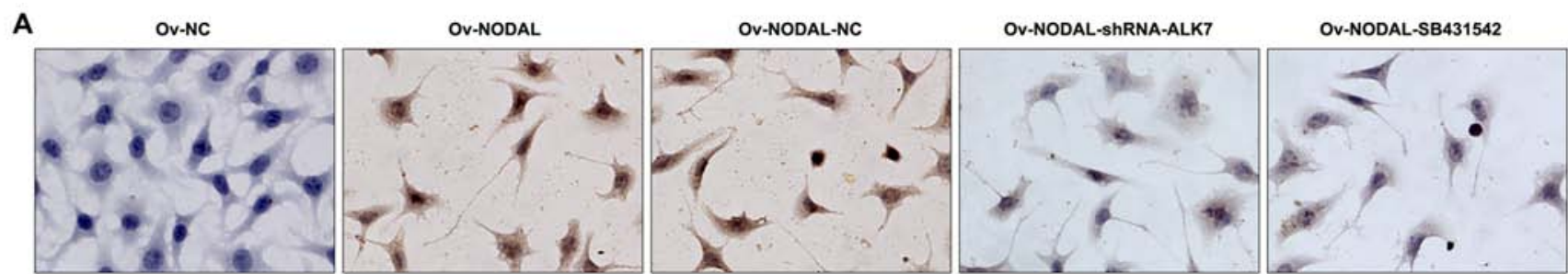

B
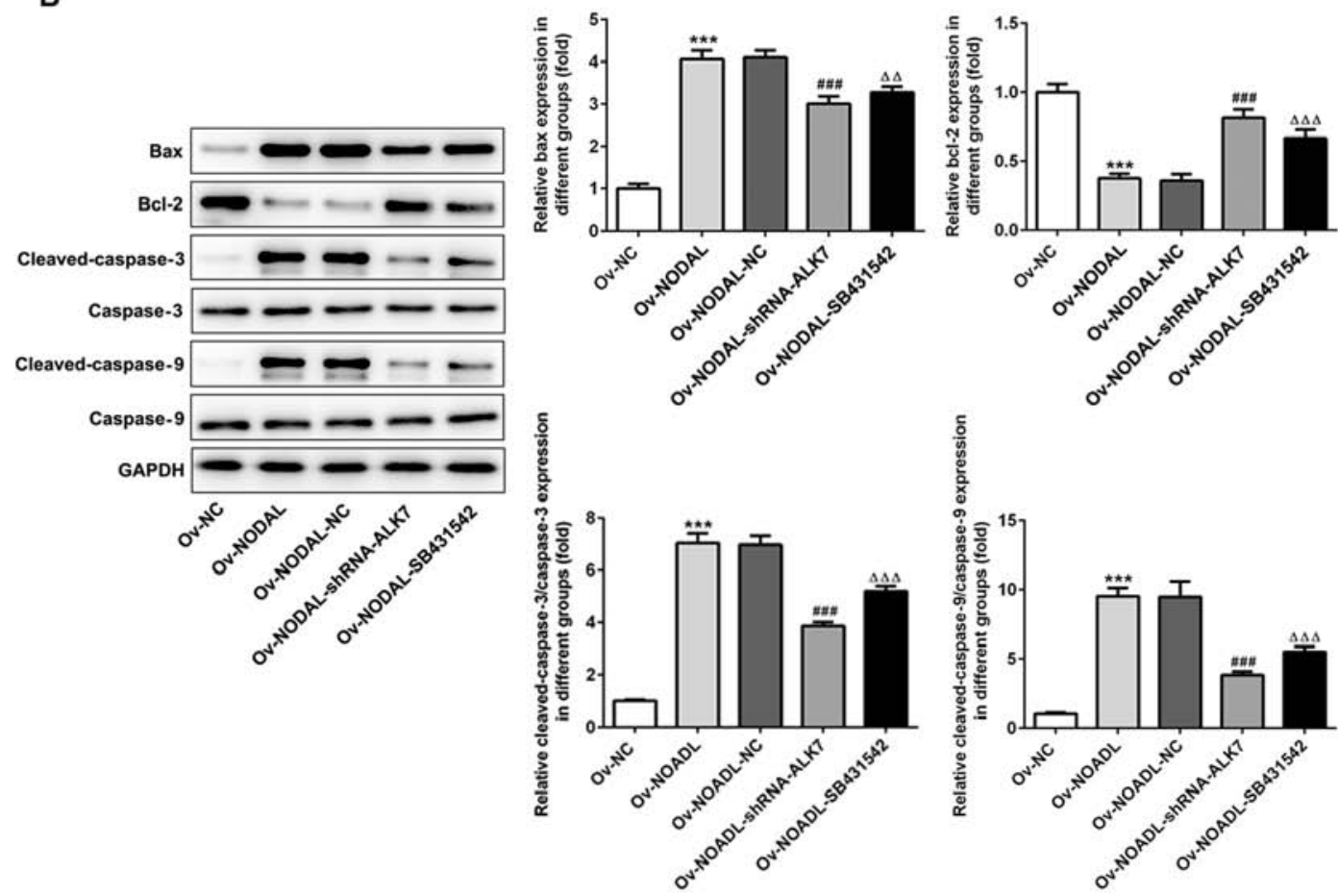

Figure 5. ALK7 inhibition reverses NODAL overexpression-mediated promotion of endometrial cancer cell apoptosis. (A) Cell apoptosis was assessed by performing TUNEL assays (magnification, $\mathrm{x} 200$ ). (B) Expression levels of apoptosis-related proteins were measured via western blotting. ${ }^{* * * *} \mathrm{P}<0.001 \mathrm{vs} \mathrm{Ov}-\mathrm{NC}$; ${ }^{\# \# \# "} \mathrm{P}<0.001$ vs. Ov-NODAL-NC; ${ }^{\Delta \Delta} \mathrm{P}<0.01$ and ${ }^{\Delta \Delta} \mathrm{P}<0.001$ vs. Ov-NODAL. ALK7, activin A receptor type 1C; NODAL, nodal growth differentiation factor; Ov, overexpression; $\mathrm{NC}$, negative control.

Li et al (28) reported that in bladder cancer tissues and cell lines, NODAL knockdown blocked the expression of ALK7. Additionally, an ALK7 inhibitor reversed the effect of NODAL overexpression on bladder cancer cell proliferation, invasion and migration. Furthermore, a previous study indicated that the overexpression of NODAL and its receptor ALK7 induced ovarian follicle cell apoptosis, indicating that the expression of NODAL and ALK7 is crucial in gynecological diseases (29). However, to the best of our knowledge, the expression of NODAL and ALK7 in EC and whether NODAL affects EC cell proliferation, invasion, migration and apoptosis has not been previously reported. The results of the present study indicated that NODAL overexpression activated ALK7, inhibited cell proliferation, invasion and migration, and promoted apoptosis in EC cells compared with Ov-NC. Additionally, ALK7 inhibition reversed NODAL overexpression-mediated inhibition of EC cell proliferation, invasion and migration, and promotion of EC cell apoptosis. Therefore, it was hypothesized that anticancer drugs targeting NODAL or ALK7 may inhibit tumor cell proliferation, invasion and migration, and induce apoptosis by activating NODAL and ALK7. The results of the present study were consistent with the results of a study conducted by Xu et al (21), which investigated NODAL and ALK7 in ovarian cancer. The aforementioned study indicated that NODAL inhibited cell proliferation and promoted cell apoptosis in ovarian cancer by activating ALK7. This finding provided a potential explanation for the occurrence of endometriosis near the ovaries of women with ovarian cancer, indicating an inextricable association between the two (30).

In the present study, ALK7 knockdown or inhibition did not completely reverse NODAL overexpression-mediated effects on EC cells. It was hypothesized that a potential reason was that NODAL acted on EC cells not only via the ALK7 signaling pathway, but also via other signaling pathways. For instance, NODAL promoted renal cell carcinoma cell proliferation by activating the Smad and ERK1/2 signaling pathways (31). Additionally, NODAL promotes non-small cell lung cancer cell malignancy via activation of the NF- $\mathrm{KB} / \mathrm{IL}-6$ signaling pathway (32). Therefore, whether NODAL acts via other signaling pathways in $\mathrm{EC}$ requires further investigation.

The present study only investigated the effect of the NODAL/ALK7 signaling pathway on EC cells. Therefore, other mechanisms underlying NODAL in EC cells should be explored in future studies. Furthermore, the present study only conducted in vitro experiments, but in vivo experiments. 
Therefore, future studies should investigate the effect of NODAL on EC with animal models.

In conclusion, the present study indicated that NODAL inhibited EC cell proliferation, invasion and migration, and promoted EC cell apoptosis, potentially via activating ALK7. Therefore, the present study identified potential novel therapeutic targets for EC.

\section{Acknowledgements}

Not applicable.

\section{Funding}

The present study was supported by the Guangdong Medical Science and Technology Research Foundation (grant no. A2018059).

\section{Availability of data and materials}

The datasets used and/or analyzed during the current study are available from the corresponding author on reasonable request.

\section{Authors' contributions}

WZ, XL and HZ conceptualized and designed the current study. XH, BW, YL and HW acquired, analyzed and interpreted data. XH, HZ, YL and HW drafted the manuscript and revised it critically for important intellectual content. All authors agreed to be held accountable for the current study in ensuring questions related to the integrity of any part of the work are appropriately investigated and resolved. All authors read approved the final manuscript.

\section{Ethics approval and consent to participate}

Not applicable.

\section{Patient consent for publication}

Not applicable.

\section{Competing interests}

The authors declare that they have no competing interests.

\section{References}

1. Zhong Y, Wang Y, Dang H and Wu X: LncRNA AFAP1-AS1 contributes to the progression of endometrial carcinoma by regulating miR-545-3p/VEGFA pathway. Mol Cell Probes 53: 101606, 2020.

2. Moore K and Brewer MA: Endometrial cancer: Is this a new disease? Am Soc Clin Oncol Educ Book 37: 435-442, 2017.

3. Lee YC, Lheureux S and Oza AM: Treatment strategies for endometrial cancer: Current practice and perspective. Curr Opin Obstet Gynecol 29: 47-58, 2017.

4. Zinski J, Tajer B and Mullins MC: TGF-beta family signaling in early vertebrate development. Cold Spring Harb Perspect Biol 10: a033274, 2018

5. Martyn I, Kanno TY, Ruzo A, Siggia ED and Brivanlou AH: Self-organization of a human organizer by combined Wnt and Nodal signalling. Nature 558: 132-135, 2018.
6. Topczewska JM, Postovit LM, Margaryan NV, Sam A, Hess AR, Wheaton WW, Nickoloff BJ, Topczewski J and Hendrix MJ: Embryonic and tumorigenic pathways converge via Nodal signaling: Role in melanoma aggressiveness. Nat Med 12: 925-932, 2006

7. Gong W, Sun B, Zhao X, Zhang D, Sun J, Liu T, Gu Q, Dong X, Liu F, Wang Y, et al: Nodal signaling promotes vasculogenic mimicry formation in breast cancer via the $\mathrm{Smad} 2 / 3$ pathway. Oncotarget 7: 70152-70167, 2016.

8. Qi YF, Wu L, Li ZQ, Wu ML, Wang HF, Chan KY, Lu LL, Cai SH, Wang HS and Du J: Nodal signaling modulates the expression of Oct- 4 via nuclear translocation of $\beta$-catenin in lung and prostate cancer cells. Arch Biochem Biophys 608: 34-41, 2016.

9. Kong B, Wang W, Esposito I, Friess H, Michalski CW and Kleeff J: Increased expression of Nodal correlates with reduced patient survival in pancreatic cancer. Pancreatology 15: 156-161, 2015.

10. Sun C, Sun L, Jiang K, Gao DM, Kang XN, Wang C, Zhang S, Huang S, Qin X, Li Y and Liu YK: NANOG promotes liver cancer cell invasion by inducing epithelial-mesenchymal transition through NODAL/SMAD3 signaling pathway. Int J Biochem Cell Biol 45: 1099-1108, 2013.

11. Perkhofer L, Walter K, Costa IG, Carrasco MC, Eiseler T, Hafner S, Genze F, Zenke M, Bergmann W, Illing A, et al: Tbx3 fosters pancreatic cancer growth by increased angiogenesis and activin/nodal-dependent induction of stemness. Stem Cell Res 17: 367-378, 2016.

12. Bodenstine TM, Chandler GS, Reed DW, Margaryan NV, Gilgur A, Atkinson J, Ahmed N, Hyser M, Seftor EA, Strizzi L and Hendrix MJ: Nodal expression in triple-negative breast cancer: Cellular effects of its inhibition following doxorubicin treatment. Cell Cycle 15: 1295-1302, 2016.

13. Lawrence MG, Margaryan NV, Loessner D, Collins A, Kerr KM, Turner M, Seftor EA, Stephens CR,Lai J; APC BioResource, et al: Reactivation of embryonic nodal signaling is associated with tumor progression and promotes the growth of prostate cancer cells. Prostate 71: 1198-1209, 2011.

14. Wakefield LM and Hill CS: Beyond TGF $\beta$ : Roles of other TGF $\beta$ superfamily members in cancer. Nat Rev Cancer 13: 328-341, 2013.

15. Inman GJ, Nicolás FJ, Callahan JF, Harling JD, Gaster LM, Reith AD, Laping NJ and Hill CS: SB-431542 is a potent and specific inhibitor of transforming growth factor-beta superfamily type I activin receptor-like kinase (ALK) receptors ALK4, ALK5, and ALK7. Mol Pharmacol 62: 65-74, 2002.

16. Roberts HJ, Hu S, Qiu Q, Leung PC, Caniggia I, Gruslin A, Tsang B and Peng C: Identification of novel isoforms of activin receptor-like kinase 7 (ALK7) generated by alternative splicing and expression of ALK7 and its ligand, Nodal, in human placenta. Biol Reprod 68: 1719-1726, 2003.

17. Hu T, Su F, Jiang W and Dart DA: Overexpression of activin receptor-like kinase 7 in breast cancer cells is associated with decreased cell growth and adhesion. Anticancer Res 37: 3441-3451, 2017.

18. Liu C, Yang Z, Li D, Liu Z, Miao X, Yang L, Zou Q and Yuan Y: Overexpression of B2M and loss of ALK7 expression are associated with invasion, metastasis, and poor-prognosis of the pancreatic ductal adenocarcinoma. Cancer Biomark 15: 735-743, 2015.

19. Michael IP, Saghafinia S, Tichet M, Zangger N, Marinoni I, Perren A and Hanahan D: ALK7 signaling manifests a homeostatic tissue barrier that is abrogated during tumorigenesis and metastasis. Dev Cell 49: 409-424.e6, 2019.

20. Zhong Y, Xu G, Ye G, Lee D, Modica-Amore J and Peng C: Nodal and activin receptor-like kinase 7 induce apoptosis in human breast cancer cell lines: Role of caspase 3. Int J Physiol Pathophysiol Pharmacol 1: 83-96, 2009.

21. Xu G, Zhong Y, Munir S, Yang BB, Tsang BK and Peng C: Nodal induces apoptosis and inhibits proliferation in human epithelial ovarian cancer cells via activin receptor-like kinase 7. J Clin Endocrinol Metab 89: 5523-5534, 2004.

22. Strizzi L, Hardy KM, Kirschmann DA, Ahrlund-Richter L and Hendrix MJ: Nodal expression and detection in cancer: Experience and challenges. Cancer Res 72: 1915-1920, 2012.

23. Venuto S, Castellana S, Monti M, Appolloni I, Fusilli C, Fusco C, Pucci P, Malatesta P, Mazza T, Merla G and Micale L: TRIM8-driven transcriptomic profile of neural stem cells identified glioma-related nodal genes and pathways. Biochim Biophys Acta Gen Subj 1863: 491-501, 2019.

24. Kantor O, Wong S, Weiss A, Metzger O, Mittendorf EA and King TA: Prognostic significance of residual nodal disease after neoadjuvant endocrine therapy for hormone receptor-positive breast cancer. NPJ Breast Cancer 6: 35, 2020. 
25. Quail DF, Walsh LA, Zhang G, Findlay SD, Moreno J, Fung L, Ablack A, Lewis JD, Done SJ, Hess DA and Postovit LM: Embryonic protein nodal promotes breast cancer vascularization. Cancer Res 72: 3851-3863, 2012.

26. Chen J, Liu WB, Jia WD, Xu GL, Ma JL, Ren Y, Chen H, Sun SN, Huang M and Li JS: Embryonic morphogen nodal is associated with progression and poor prognosis of hepatocellular carcinoma. PLoS One 9: e85840, 2014

27. Munir S, Xu G, Wu Y, Yang B, Lala PK and Peng C: Nodal and ALK7 inhibit proliferation and induce apoptosis in human trophoblast cells. J Biol Chem 279: 31277-31286, 2004.

28. Li Y, Zhong W, Zhu M, Hu S and Su X: Nodal regulates bladder cancer cell migration and invasion via the ALK/Smad signaling pathway. Onco Targets Ther 11: 6589-6597, 2018.

29. Wang H, Jiang JY, Zhu C, Peng C and Tsang BK: Role and regulation of nodal/activin receptor-like kinase 7 signaling pathway in the control of ovarian follicular atresia. Mol Endocrinol 20 2469-2482, 2006.
30. Ruderman R and Pavone ME: Ovarian cancer in endometriosis: An update on the clinical and molecular aspects. Minerva Ginecol 69: 286-294, 2017.

31. Zhang Z, Jiang T, Li Q, Wang J, Yang D, Li X, Wang Q and Song X: Nodal activates smad and extracellular signal-regulated kinases $1 / 2$ pathways promoting renal cell carcinoma proliferation. Mol Med Rep 12: 587-594, 2015.

32. Xu X, Zhou X, Gao C, Cao L, Zhang Y, Hu X and Cui Y: Nodal promotes the malignancy of non-small cell lung cancer (NSCLC) cells via activation of NF- $\kappa \mathrm{B} / \mathrm{IL}-6$ signals. Biol Chem 400: 777-785, 2019.

This work is licensed under a Creative Commons Attribution-NonCommercial-NoDerivatives 4.0 International (CC BY-NC-ND 4.0) License. 